\title{
An implicit method for finding a common fixed point of a representation of nonexpansive mappings in Banach spaces
}

\author{
Nawab Hussain ${ }^{1}$, Mahmood Lashkarizadeh Bami ${ }^{2}$ and Ebrahim Soori ${ }^{3^{*}}$
}

"Correspondence: sori.e@lu.ac.ir; Sori.ebrahim@yahoo.com ${ }^{3}$ Department of Mathematics, Lorestan University, P.O. Box 465 , Khoramabad, Lorestan, Iran Full list of author information is available at the end of the article

\begin{abstract}
We introduce an implicit method for finding an element of the set of common fixed points of a representation of nonexpansive mappings. Then we prove the strong convergence of the proposed implicit scheme to the common fixed point of a representation of nonexpansive mappings.
\end{abstract}

MSC: $90 \mathrm{C} 33 ; 47 \mathrm{H} 10$

Keywords: fixed point; nonexpansive mapping; representation; semigroup; sunny nonexpansive retraction

\section{Introduction}

Let $C$ be a nonempty closed and convex subset of a Banach space $E$ and $E^{*}$ be the dual space of $E$. Let $\langle\cdot, \cdot\rangle$ denote the pairing between $E$ and $E^{*}$. The normalized duality mapping $J: E \rightarrow E^{*}$ is defined by

$$
J(x)=\left\{f \in E^{*}:\langle x, f\rangle=\|x\|^{2}=\|f\|^{2}\right\}
$$

for all $x \in E$. In the sequel, we use $j$ to denote the single-valued normalized duality mapping. Let $U=\{x \in E:\|x\|=1\}$. $E$ is said to be smooth or to have a Gâteaux differentiable norm if the limit

$$
\lim _{t \rightarrow 0} \frac{\|x+t y\|-\|x\|}{t}
$$

exists for each $x, y \in U$. $E$ is said to have a uniformly Gâteaux differentiable norm if for each $y \in U$, the limit is attained uniformly for all $x \in U$. $E$ is said to be uniformly smooth or is said to have a uniformly Féchet differentiable norm if the limit is attained uniformly for $x, y \in U$. It is known that if the norm of $E$ is uniformly Gâteaux differentiable, then the duality mapping $J$ is single-valued and uniformly norm to weak* continuous on each bounded subset of $E$. A Banach space $E$ is smooth if the duality mapping $J$ of $E$ is singlevalued. We know that if $E$ is smooth, then $J$ is norm to weak-star continuous; for more details, see [1].

Let $C$ be a nonempty closed and convex subset of a Banach space $E$. A mapping $T$ of $C$ into itself is called nonexpansive if $\|T x-T y\| \leq\|x-y\|$ for all $x, y \in C$, and a mapping $f$ is an $\alpha$-contraction on $E$ if $\|f(x)-f(y)\| \leq \alpha\|x-y\|, x, y \in E$ such that $0 \leq \alpha<1$.

○2014 Hussain et al.; licensee Springer. This is an Open Access article distributed under the terms of the Creative Commons Attribution License (http://creativecommons.org/licenses/by/2.0), which permits unrestricted use, distribution, and reproduction in any medium, provided the original work is properly cited. 
In this paper, motivated by Lashkarizadeh Bami and Soori [2] and Hussain and Takahashi [3], we introduce the following general implicit algorithm for finding a common element of the set of fixed points of a representation $\mathcal{S}=\left\{T_{t}: t \in S\right\}$ of a semigroup $S$ as nonexpansive mappings from $C$ into itself, with respect to a left regular sequence of means defined on an appropriate subspace of bounded real-valued functions of the semigroup. On the other hand, our goal is to prove that there exists a sunny nonexpansive retraction $P$ of $C$ onto $\operatorname{Fix}(\mathcal{S})$ and $x \in C$ such that the following sequence $\left\{z_{n}\right\}$ converges strongly to $P x$ :

$$
z_{n}=\epsilon_{n} f\left(z_{n}\right)+\left(1-\epsilon_{n}\right) T_{\mu_{n}} z_{n} \quad(n \in \mathbb{N}) .
$$

\section{Preliminaries}

Let $S$ be a semigroup. We denote by $B(S)$ the Banach space of all bounded real-valued functions defined on $S$ with supremum norm. For each $s \in S$ and $f \in B(S)$, we define $l_{s}$ and $r_{s}$ in $B(S)$ by

$$
\left(l_{s} f\right)(t)=f(s t), \quad\left(r_{s} f\right)(t)=f(t s) \quad(t \in S)
$$

Let $X$ be a subspace of $B(S)$ containing 1 , and let $X^{*}$ be its topological dual. An element $\mu$ of $X^{*}$ is said to be a mean on $X$ if $\|\mu\|=\mu(1)=1$. We often write $\mu_{t}(f(t))$ instead of $\mu(f)$ for $\mu \in X^{*}$ and $f \in X$. Let $X$ be left invariant (resp. right invariant), i.e., $l_{s}(X) \subset X$ (resp. $\left.r_{s}(X) \subset X\right)$ for each $s \in S$. A mean $\mu$ on $X$ is said to be left invariant (resp. right invariant) if $\mu\left(l_{s} f\right)=\mu(f)$ (resp. $\left.\mu\left(r_{s} f\right)=\mu(f)\right)$ for each $s \in S$ and $f \in X . X$ is said to be left (resp. right) amenable if $X$ has a left (resp. right) invariant mean. $X$ is amenable if $X$ is both left and right amenable. As is well known, $B(S)$ is amenable when $S$ is a commutative semigroup (see p.29 of [1]). A net $\left\{\mu_{\alpha}\right\}$ of means on $X$ is said to be left regular if

$$
\lim _{\alpha}\left\|l_{s}^{*} \mu_{\alpha}-\mu_{\alpha}\right\|=0
$$

for each $s \in S$, where $l_{s}^{*}$ is the adjoint operator of $l_{s}$.

Let $f$ be a function of the semigroup $S$ into a reflexive Banach space $E$ such that the weak closure of $\{f(t): t \in S\}$ is weakly compact, and let $X$ be a subspace of $B(S)$ containing all the functions $t \rightarrow\left\langle f(t), x^{*}\right\rangle$ with $x^{*} \in E^{*}$. We know from [4] that for any $\mu \in X^{*}$, there exists a unique element $f_{\mu}$ in $E$ such that $\left\langle f_{\mu}, x^{*}\right\rangle=\mu_{t}\left\langle f(t), x^{*}\right\rangle$ for all $x^{*} \in E^{*}$. We denote such $f_{\mu}$ by $\int f(t) \mathrm{d} \mu(t)$. Moreover, if $\mu$ is a mean on $X$, then from [5], $\int f(t) \mathrm{d} \mu(t) \in \overline{c o}\{f(t): t \in S\}$.

Let $C$ be a nonempty closed and convex subset of $E$. Then a family $\mathcal{S}=\left\{T_{s}: s \in S\right\}$ of mappings from $C$ into itself is said to be a representation of $S$ as a nonexpansive mapping on $C$ into itself if $\mathcal{S}$ satisfies the following:

(1) $T_{s t} x=T_{s} T_{t} x$ for all $s, t \in S$ and $x \in C$;

(2) for every $s \in S$, the mapping $T_{s}: C \rightarrow C$ is nonexpansive.

We denote by $\operatorname{Fix}(\mathcal{S})$ the set of common fixed points of $\mathcal{S}$, that is, $\operatorname{Fix}(\mathcal{S})=\bigcap_{s \in S}\{x \in C$ : $\left.T_{s} x=x\right\}$.

Theorem 2.1 [6] Let $S$ be a semigroup, let $C$ be a closed, convex subset of a reflexive Banach space $E, \mathcal{S}=\left\{T_{s}: s \in S\right\}$ be a representation of $S$ as a nonexpansive mapping from $C$ into itself such that weak closure of $\left\{T_{t} x: t \in S\right\}$ is weakly compact for each $x \in C$, and let $X$ be a subspace of $B(S)$ such that $1 \in X$ and the mapping $t \rightarrow\left\langle T(t) x, x^{*}\right\rangle$ be an element of $X$ for 
each $x \in C$ and $x^{*} \in E$, and $\mu$ be a mean on $X$. If we write $T_{\mu} x$ instead of $\int T_{t} x \mathrm{~d} \mu(t)$, then the following hold.

(i) $T_{\mu}$ is a nonexpansive mapping from $C$ into $C$.

(ii) $T_{\mu} x=x$ for each $x \in \operatorname{Fix}(\mathcal{S})$.

(iii) $T_{\mu} x \in \overline{\operatorname{co}}\left\{T_{t} x: t \in S\right\}$ for each $x \in C$.

(iv) If $X$ is $r_{s}$-invariant for each $s \in S$ and $\mu$ is right invariant, then $T_{\mu} T_{t}=T_{\mu}$ for each $t \in S$.

Remark From Theorem 4.1.6 in [1], every uniformly convex Banach space is strictly convex and reflexive.

Let $D$ be a subset of $B$, where $B$ is a subset of a Banach space $E$, and let $P$ be a retraction of $B$ onto $D$, that is, $P x=x$ for each $x \in D$. Then $P$ is said to be sunny if for each $x \in B$ and $t \geq 0$ with $P x+t(x-P x) \in B, P(P x+t(x-P x))=P x$. A subset $D$ of $B$ is said to be a sunny nonexpansive retract of $B$ if there exists a sunny nonexpansive retraction $P$ of $B$ onto $D$. We know that if $E$ is smooth and $P$ is a retraction of $B$ onto $D$, then $P$ is sunny and nonexpansive if and only if for each $x \in B$ and $z \in D,\langle x-P x, J(z-P x)\rangle \leq 0$. For more details, see [1].

Lemma 2.2 [7] Let $S$ be a semigroup, and let $C$ be a compact convex subset of a real strictly convex and smooth Banach space E. Suppose that $\mathcal{S}=\left\{T_{s}: s \in S\right\}$ is a representation of $S$ as a nonexpansive mapping from $C$ into itself. Let $X$ be a left invariant subspace of $B(S)$ such that $1 \in X$, and the function $t \mapsto\left\langle T_{t} x, x^{*}\right\rangle$ is an element of $X$ for each $x \in C$ and $x^{*} \in E^{*}$. If $\mu$ is a left invariant mean on $X$, then $\operatorname{Fix}\left(T_{\mu}\right)=T_{\mu} C=\operatorname{Fix}(\mathcal{S})$ and there exists a unique sunny nonexpansive retraction from $C$ onto $\operatorname{Fix}(\mathcal{S})$.

Throughout the rest of this paper, the open ball of radius $r$ centered at 0 is denoted by $B_{r}$. Let $C$ be a nonempty closed convex subset of a Banach space $E$. For $\epsilon>0$ and a mapping $T: C \rightarrow C$, we let $F_{\epsilon}(T)$ be the set of $\epsilon$-approximate fixed points of $T$, i.e., $F_{\epsilon}(T)=\{x \in C$ : $\|x-T x\| \leq \epsilon\}$.

\section{Main result}

In this section, we deal with a strong convergence approximation scheme for finding a common element of the set of common fixed points of a representation of nonexpansive mappings.

Theorem 3.1 Let $S$ be a semigroup. Let $C$ be a nonempty compact convex subset of a real strictly convex and reflexive and smooth Banach space E. Suppose that $\mathcal{S}=\left\{T_{s}: s \in S\right\}$ is a representation of $S$ as a nonexpansive mapping from $C$ into itself such that $\operatorname{Fix}(\mathcal{S}) \neq \emptyset$. Let $X$ be a left invariant subspace of $B(S)$ such that $1 \in X$, and the function $t \mapsto\left\langle T_{t} x, x^{*}\right\rangle$ is an element of $X$ for each $x \in C$ and $x^{*} \in E^{*}$. Let $\left\{\mu_{n}\right\}$ be a left regular sequence of means on $X$. Suppose that $f$ is an $\alpha$-contraction on C. Let $\epsilon_{n}$ be a sequence in $(0,1)$ such that $\lim _{n} \epsilon_{n}=0$. Then there exists a unique sunny nonexpansive retraction $P$ of $C$ onto $\operatorname{Fix}(\mathcal{S})$ and $x \in C$ such that the following sequence $\left\{z_{n}\right\}$ generated by

$$
z_{n}=\epsilon_{n} f\left(z_{n}\right)+\left(1-\epsilon_{n}\right) T_{\mu_{n}} z_{n} \quad(n \in \mathbb{N})
$$

strongly converges to $P x$. 
Proof By Proposition 1.7.3 and Theorem 1.9.21 in [8], any compact subset $C$ of a reflexive Banach space $E$ is weakly compact, and from Proposition 1.9.18 in [8], any closed convex subset of a weakly compact subset $C$ of a Banach space $E$ is itself weakly compact, and by Proposition 1.9.13 in [8], any convex subset $C$ of a normed space $E$ is weakly closed if and only if $C$ is closed. Therefore, weak closure of $\left\{T_{t} x: t \in S\right\}$ is weakly compact for each $x \in C$.

We divide the proof into five steps.

Step 1 . The existence of $z_{n}$ which satisfies (1).

This follows immediately from the fact that for every $n \in \mathbb{N}$, the mapping $N_{n}$ given by

$$
N_{n} x:=\epsilon_{n} f(x)+\left(1-\epsilon_{n}\right) T_{\mu_{n}} x \quad(x \in C)
$$

is a contraction. To see this, put $\beta_{n}=\left(1+\epsilon_{n}(\alpha-1)\right)$, then $0 \leq \beta_{n}<1(n \in \mathbb{N})$. Then we have

$$
\begin{aligned}
\left\|N_{n} x-N_{n} y\right\| & \leq \epsilon_{n}\|f(x)-f(y)\|+\left(1-\epsilon_{n}\right)\left\|T_{\mu_{n}} x-T_{\mu_{n}} y\right\| \\
& \leq \epsilon_{n} \alpha\|x-y\|+\left(1-\epsilon_{n}\right)\|x-y\| \\
& =\left(1+\epsilon_{n}(\alpha-1)\right)\|x-y\|=\beta_{n}\|x-y\| .
\end{aligned}
$$

Therefore, by the Banach contraction principle [1], there exists a unique point $z_{n} \in C$ such that $N_{n} z_{n}=z_{n}$.

Step 2. $\lim _{n \rightarrow \infty}\left\|z_{n}-T_{t} z_{n}\right\|=0$ for all $t \in S$.

Consider $t \in S$ and let $\epsilon>0$. By Lemma 1 in [9], there exists $\delta>0$ such that $\overline{\mathrm{co}} F_{\delta}\left(T_{t}\right)+$ $2 B_{\delta} \subseteq F_{\epsilon}\left(T_{t}\right)$. By Corollary 2.8 in [10], there also exists a natural number $N$ such that

$$
\left\|\frac{1}{N+1} \sum_{i=0}^{N} T_{t^{i} s} y-T_{t}\left(\frac{1}{N+1} \sum_{i=0}^{N} T_{t^{i} s} y\right)\right\| \leq \delta
$$

for all $s \in S$ and $y \in C$. Let $p \in \operatorname{Fix}(\mathcal{S})$ and $M_{0}$ be a positive number such that $\sup _{y \in C}\|y\| \leq$ $M_{0}$. Let $t \in S$, since $\left\{\mu_{n}\right\}$ is strongly left regular, there exists $N_{0} \in \mathbb{N}$ such that $\left\|\mu_{n}-l_{t^{i}}^{*} \mu_{n}\right\| \leq$ $\frac{\delta}{\left(3 M_{0}\right)}$ for $n \geq N_{0}$ and $i=1,2, \ldots, N$. Then we have

$$
\begin{aligned}
& \sup _{y \in C}\left\|T_{\mu_{n}} y-\int \frac{1}{N+1} \sum_{i=0}^{N} T_{t^{i} s} y \mathrm{~d} \mu_{n}(s)\right\| \\
& =\sup _{y \in C} \sup _{\left\|x^{*}\right\|=1}\left|\left\langle T_{\mu_{n}} y, x^{*}\right\rangle-\left\langle\int \frac{1}{N+1} \sum_{i=0}^{N} T_{t^{i} s} y \mathrm{~d} \mu_{n}(s), x^{*}\right\rangle\right| \\
& =\sup _{y \in C} \sup _{\left\|x^{*}\right\|=1}\left|\frac{1}{N+1} \sum_{i=0}^{N}\left(\mu_{n}\right)_{s}\left\langle T_{s} y, x^{*}\right\rangle-\frac{1}{N+1} \sum_{i=0}^{N}\left(\mu_{n}\right)_{s}\left\langle T_{t^{i} s} y, x^{*}\right\rangle\right| \\
& \leq \frac{1}{N+1} \sum_{i=0}^{N} \sup _{y \in C} \sup _{\left\|x^{*}\right\|=1}\left|\left(\mu_{n}\right)_{s}\left\langle T_{s} y, x^{*}\right\rangle-\left(l_{t^{i}}^{*} \mu_{n}\right)_{s}\left\langle T_{s} y, x^{*}\right\rangle\right| \\
& \leq \max _{i=1,2, \ldots, N}\left\|\mu_{n}-l_{t^{i}}^{*} \mu_{n}\right\|\left(M_{0}+2\|p\|\right) \\
& \leq \max _{i=1,2, \ldots, N}\left\|\mu_{n}-l_{t^{i}}^{*} \mu_{n}\right\|\left(3 M_{0}\right) \\
& \leq \delta \quad\left(n \geq N_{0}\right) .
\end{aligned}
$$


By Theorem 2.1 we have

$$
\int \frac{1}{N+1} \sum_{i=0}^{N} T_{t^{i} s} y \mathrm{~d} \mu_{n}(s) \in \overline{\mathrm{co}}\left\{\frac{1}{N+1} \sum_{i=0}^{N} T_{t^{i}}\left(T_{s} y\right): s \in S\right\} .
$$

It follows from (2)-(4) that

$$
\begin{gathered}
T_{\mu_{n}} y \in \overline{\mathrm{co}}\left\{\frac{1}{N+1} \sum_{i=0}^{N} T_{t^{i} s} y: s \in S\right\}+B_{\delta} \\
\subset \overline{\operatorname{co}} F_{\delta}\left(T_{t}\right)+2 B_{\delta} \subset F_{\epsilon}\left(T_{t}\right)
\end{gathered}
$$

for all $y \in C$ and $n \geq N_{0}$. Therefore, $\limsup _{n \rightarrow \infty} \sup _{y \in C}\left\|T_{t}\left(T_{\mu_{n}} y\right)-T_{\mu_{n}} y\right\| \leq \epsilon$. Since $\epsilon>0$ is arbitrary, we have

$$
\limsup _{n \rightarrow \infty} \sup _{y \in C}\left\|T_{t}\left(T_{\mu_{n}} y\right)-T_{\mu_{n}} y\right\|=0
$$

Let $t \in S$ and $\epsilon>0$, then there exists $\delta>0$ which satisfies (2). Take $L_{0}=(1+\alpha) 2 M_{0}+$ $\|f(p)-p\|$. Now, from the condition $\lim _{n} \epsilon_{n}=0$ and from (5), there exists a natural number $N_{1}$ such that $T_{\mu_{n}} y \in F_{\delta}\left(T_{t}\right)$ for all $y \in C$ and $\epsilon_{n}<\frac{\delta}{2 L_{0}}$ for all $n \geq N_{1}$. Since $p \in \operatorname{Fix}(\mathcal{S})$, we have

$$
\begin{aligned}
\epsilon_{n}\left\|f\left(z_{n}\right)-T_{\mu_{n}} z_{n}\right\| & \leq \epsilon_{n}\left(\left\|f\left(z_{n}\right)-f(p)\right\|+\|f(p)-p\|+\left\|T_{\mu_{n}} p-T_{\mu_{n}} z_{n}\right\|\right) \\
& \leq \epsilon_{n}\left(\alpha\left\|z_{n}-p\right\|+\|f(p)-p\|+\|A\|\left\|z_{n}-p\right\|\right) \\
& \leq \epsilon_{n}\left(\alpha\left\|z_{n}-p\right\|+\|f(p)-p\|+\left\|z_{n}-p\right\|\right) \\
& \leq \epsilon_{n}\left((1+\alpha)\left\|z_{n}-p\right\|+\|f(p)-p\|\right) \\
& \leq \epsilon_{n}\left((1+\alpha) 2 M_{0}+\|f(p)-p\|\right) \\
& =\epsilon_{n} L_{0} \leq \frac{\delta}{2}
\end{aligned}
$$

for all $n \geq N_{1}$. Observe that

$$
\begin{aligned}
z_{n} & =\epsilon_{n} f\left(z_{n}\right)+\left(1-\epsilon_{n}\right) T_{\mu_{n}} z_{n} \\
& =T_{\mu_{n}} z_{n}+\epsilon_{n}\left(f\left(z_{n}\right)-T_{\mu_{n}} z_{n}\right) \\
& \in F_{\delta}\left(T_{t}\right)+B_{\frac{\delta}{2}} \\
& \subseteq F_{\delta}\left(T_{t}\right)+2 B_{\delta} \\
& \subseteq F_{\epsilon}\left(T_{t}\right)
\end{aligned}
$$

for all $n \geq N_{1}$. This shows that

$$
\left\|z_{n}-T_{t} z_{n}\right\| \leq \epsilon \quad\left(n \geq N_{1}\right)
$$

Since $\epsilon>0$ is arbitrary, we get $\lim _{n \rightarrow \infty}\left\|z_{n}-T_{t} z_{n}\right\|=0$. 
Step 3. $\mathfrak{S}\left\{z_{n}\right\} \subset \operatorname{Fix}(\mathcal{S})$, where $\mathfrak{S}\left\{z_{n}\right\}$ denotes the set of strongly limit points of $\left\{z_{n}\right\}$.

Let $z \in \mathfrak{S}\left\{z_{n}\right\}$, and let $\left\{z_{n_{j}}\right\}$ be a subsequence of $\left\{z_{n}\right\}$ such that $z_{n_{j}} \rightarrow z$,

$$
\begin{aligned}
\left\|T_{t} z-z\right\| & \leq\left\|T_{t} z-T_{t} z_{n_{j}}\right\|+\left\|T_{t} z_{n_{j}}-z_{n_{j}}\right\|+\left\|z_{n_{j}}-z\right\| \\
& \leq 2\left\|z_{n_{j}}-z\right\|+\left\|T_{t} z_{n_{j}}-z_{n_{j}}\right\|,
\end{aligned}
$$

then by Step 2,

$$
\left\|T_{t} z-z\right\| \leq 2 \lim _{j}\left\|z_{n_{j}}-z\right\|+\lim _{j}\left\|T_{t} z_{n_{j}}-z_{n_{j}}\right\|=0,
$$

therefore $z \in \operatorname{Fix}(\mathcal{S})$.

Step 4. There exists a unique sunny nonexpansive retraction $P$ of $C$ onto $\operatorname{Fix}(\mathcal{S})$ and $x \in C$ such that

$$
\Gamma:=\limsup _{n}\left\langle x-P x, J\left(z_{n}-P x\right)\right\rangle \leq 0 .
$$

By Lemma 2.2 there exists a unique sunny nonexpansive retraction $P$ of $C$ onto $\operatorname{Fix}(\mathcal{S})$. The Banach contraction mapping principle guarantees that $f P$ has a unique fixed point $x \in C$. We show that

$$
\Gamma:=\limsup _{n}\left\langle x-P x, J\left(z_{n}-P x\right)\right\rangle \leq 0
$$

Note that from the definition of $\Gamma$ and the fact that $C$ is a compact subset of $E$, we can select a subsequence $\left\{z_{n_{j}}\right\}$ of $\left\{z_{n}\right\}$ with the following properties:

(i) $\lim _{j}\left\langle x-P x, J\left(z_{n_{j}}-P x\right)\right\rangle=\Gamma$;

(ii) $\left\{z_{n_{j}}\right\}$ converges strongly to a point $z$.

By Step 3, we have $z \in \operatorname{Fix}(\mathcal{S})$. Since $E$ is smooth, we have

$$
\Gamma=\lim _{j}\left\langle x-P x, J\left(z_{n_{j}}-P x\right)\right\rangle=\langle x-P x, J(z-P x)\rangle \leq 0 .
$$

Since $f P x=x$, we have $(f-I) P x=x-P x$. From Theorem 4.2.1(v) in [1], for $x, y \in E$ and $f \in J(y),\|x\|^{2}-\|y\|^{2} \geq 2(x-y, f)$. Therefore, for each $n \in \mathbb{N}$, we have

$$
\begin{aligned}
& \epsilon_{n}(\alpha-1)\left\|z_{n}-P x\right\|^{2} \\
& \quad \geq\left[\epsilon_{n} \alpha\left\|z_{n}-P x\right\|+\left(1-\epsilon_{n}\right)\left\|z_{n}-P x\right\|\right]^{2}-\left\|z_{n}-P x\right\|^{2} \\
& \quad \geq {\left[\epsilon_{n}\left\|f\left(z_{n}\right)-f(P x)\right\|+\left(1-\epsilon_{n}\right)\left\|T_{\mu_{n}} z_{n}-P x\right\|\right]^{2}-\left\|z_{n}-P x\right\|^{2} } \\
& \quad \geq 2\left\langle\epsilon_{n}\left(f\left(z_{n}\right)-f(P x)\right)+\left(1-\epsilon_{n}\right)\left(T_{\mu_{n}} z_{n}-P x\right)-\left(z_{n}-P x\right), J\left(z_{n}-P x\right)\right\rangle \\
& \quad=-2 \epsilon_{n}\left\langle(f-I) P x, J\left(z_{n}-P x\right)\right\rangle \\
& \quad=-2 \epsilon_{n}\left\langle x-P x, J\left(z_{n}-P x\right)\right\rangle .
\end{aligned}
$$

Hence

$$
\left\|z_{n}-P x\right\|^{2} \leq \frac{2}{1-\alpha}\left\langle x-P x, J\left(z_{n}-P x\right)\right\rangle .
$$


Step 5. $\left\{z_{n}\right\}$ strongly converges to $P x$.

Indeed, from (6), (7) and $P x \in \operatorname{Fix}(\mathcal{S})$, we conclude

$$
\limsup _{n}\left\|z_{n}-P x\right\|^{2} \leq \frac{2}{1-\alpha} \limsup _{n}\left\langle x-P x, J\left(z_{n}-P x\right)\right\rangle \leq 0 .
$$

That is, $z_{n} \rightarrow P x$.

Remark 3.2 It would be an interesting problem to prove Theorem 3.1 for continuous representations instead of nonexpansive.

\section{Competing interests}

The authors declare that they have no competing interests.

\section{Authors' contributions}

All authors contributed equally and significantly in writing this article. All authors read and approved the final manuscript.

\section{Author details}

'Department of Mathematics, King Abdulaziz University, P.O. Box 80203, Jeddah, 21589, Saudi Arabia. ${ }^{2}$ Department of Mathematics, University of Isfahan, Isfahan, Iran. ${ }^{3}$ Department of Mathematics, Lorestan University, P.O. Box 465,

Khoramabad, Lorestan, Iran.

\section{Acknowledgements}

This article was funded by the Deanship of Scientific Research (DSR), King Abdulaziz University, Jeddah. The first author acknowledges with thanks DSR, KAU for financial support. The authors would like to thank the referee of the paper for his helpful comments and invaluable suggestions. This research was supported by the Center of Excellence for Mathematics and the Office of Graduate Studies of the Lorestan University and the University of Isfahan.

Received: 31 August 2014 Accepted: 12 November 2014 Published: 04 Dec 2014

\section{References}

1. Takahashi, W: Nonlinear Functional Analysis: Fixed Point Theory and Its Applications. Yokohama Publishers, Yokohama (2000)

2. Lashkarizadeh Bami, M, Soori, E: Strong convergence of a general implicit algorithm for variational inequality problems and equilibrium problems and a continuous representation of nonexpansive mappings. Bull. Iran. Math. Soc. 40, 977-1001 (2014)

3. Hussain, $\mathrm{N}$, Takahashi, W: Weak and strong convergence theorems for semigroups of mappings without continuity in Hilbert spaces. J. Nonlinear Convex Anal. 14(4), 769-783 (2013)

4. Hirano, N, Kido, K, Takahashi, W: Nonexpansive retractions and nonlinear ergodic theorems in Banach spaces. Nonlinear Anal. 12, 1269-1281 (1988)

5. Kido, K, Takahashi, W: Mean ergodic theorems for semigroups of linear operators. J. Math. Anal. Appl. 103, 387-394 (1984)

6. Saeidi, S: Existence of ergodic retractions for semigroups in Banach spaces. Nonlinear Anal. 69, 3417-3422 (2008)

7. Saeidi, S: A note on 'Approximation of fixed points for amenable semigroups of nonexpansive mappings in Banach spaces'. Nonlinear Anal. 72, 546-548 (2010)

8. Agarwal, RP, Oregan, D, Sahu, DR: Fixed Point Theory for Lipschitzian-Type Mappings with Applications. Topological Fixed Point Theory and Its Applications, vol. 6. Springer, New York (2009)

9. Shioji, N, Takahashi, W: Strong convergence of averaged approximants for asymptotically nonexpansive mappings in Banach spaces. J. Approx. Theory 97, 53-64 (1999)

10. Atsushiba, S, Takahashi, W: A nonlinear ergodic theorem for nonexpansive mappings with compact domain. Math. Jpn. 52, 183-195 (2000)

10.1186/1687-1812-2014-238

Cite this article as: Hussain et al.: An implicit method for finding a common fixed point of a representation of nonexpansive mappings in Banach spaces. Fixed Point Theory and Applications 2014, 2014:238 